\title{
An Analysis of the Performance Determinants of Modern Pentathlon Athletes in Laser-run, A Newly-Combined Event in Modern Pentathlon
}

\author{
Chang-Hyun Lim¹, Jae-Ryang Yoon ${ }^{1}$, Chang-Soon Jeong ${ }^{2}$, Young-Sun Kim² \\ ${ }^{1}$ Human Physiology, Korea National Sport University, Seoul; ${ }^{2}$ Department of Modern Pentathlon, Korea National Sport University, Seoul, Korea
}

PURPOSE: Modern pentathlon, a combined athletic event, where final scores represent the aggregate of scores from swimming, fencing, horseback riding, and laser-run (running and shooting combined), requires participating athletes to have a wide range of athletic prowess, including cardiovascular endurance, agility, muscle power, and muscle strength. Thus, this study analyzed the predominant determinants of athletic performance in the new laser-run event by comparing elite and non-elite athletes.

METHODS: Athletes were divided into two groups, elite athletes $(\mathrm{n}=7,19.33 \pm 0.52$ years, $\mathrm{BMI}=22.18 \pm 0.59)$ and non-elite athletes $(\mathrm{n}=7,19.33 \pm 0.82$ years, $\mathrm{BMI}=22.27 \pm 1.1)$. For each athlete, a range of metabolic parameters were measured with a wireless respiratory gas analyzer during conducting laser-run. We also determined the overall performance in the laser-run event, including shooting time, accuracy, delay per shooting attempt, and running speed for each phase.

RESULTS: The elite group took significantly less time to complete the event than the non-elite group (11"47'13, and 12'20'17, respectively; $p=.015)$. Data revealed that even in shooting and running, most elements have an association with athletic performance, but running time and speed during each phase and overall showed a significant difference between groups $(p<.05)$.

CONCLUSIONS: Our data indicate that determining factors in the shooting and running phase have a close relationship with overall event performance. However, running time and speed during the running phase rather than the shooting phase were identified as being the most important determinants. Thus, reducing the time lapse during the running phase by improving cardiovascular endurance and endurance exercise capacity is highly recommended.

Key words: Modern pentathlon, Elite athlete, Laser-run, Combined event, Performance determinants

\section{INTRODUCTION}

Modern pentathlon is a combined athletic game in which final scores represent an aggregate of individual event scores from fencing, swimming, horseback riding, pistol shooting, and running. This game was initiated in ancient Greece with the purpose of testing the general physical strengths of Greek soldiers [1]. However, since the 5th Olympic Games, hosted in Stockholm in 1912, the pentathlon has become a regular event. Later, in 2009, the Union Internationale de Pentathlon Modern modi- fied the rules of the game, combining shooting and running to form a single event. Thus, the final results of an athlete's performance were to be evaluated based on the score of the combined event after fencing, swimming, and horseback riding had concluded [2]. In 2009, the original pistol used during the shooting phase, which used lead bullets, was replaced with a laser pistol. Later, in 2017, the official name of the game was changed from a combined event to "laser-run." Laser-run employs a handicapped start, which makes each athlete start 1 second later per point in order of their scores from the 3 previous events. Each athlete is

Corresponding author: Young-Sun Kim Tel +82-2-410-6693 Fax +82-2-410-6945 E-mail kim-ys62aknsu.ac.kr

*This work was supported by the Korea National Sport University.

Received 8 Jan 2018 Revised 24 Jan 2018 Accepted 4 Feb 2018

(a) This is an Open Access article distributed under the terms of the Creative Commons Attribution Non-Commercial License (http://creativecommons.org/licenses/by-nc/4.0/) which permits unrestricted non-commercial use, distribution, and reproduction in any medium, provided the original work is properly cited. 
required to participate in the shooting and running phases 4 times, hitting the targets 5 times in each shooting phase, and then run an $800 \mathrm{~m}$ course in each running phase; altogether, an athlete should attempt 20 shots and run 3,200 m. In each shooting phase, athletes should finish their 5 shots within 50 seconds for them to proceed to the next phase with 5 successful shots. If they do not hit the target 5 times successfully, athletes should then proceed to the next phase without additional penalty [2]. Laser-run is one of the most important phases in determining the outcome of the modern pentathlon event [3]. However, the newly adopted rules of the game have not yet been analyzed through detailed research. Consequently, determining the important elements that influence athletic performance is important in improving athletic performance in this new event that requires an athlete to perform effectively in both shooting and running.

Previous research by Le Meur et al. [4] revealed that overall performance in the laser-run event depends on shooting factors rather than running speed and reported that when they divided athletes into three groups based on their performance, they found no difference in time required in running between the highest-level group and the other two groups, but did find that the highest-level group took the shortest time in shooting with the least delay per shooting attempt. These authors also reported that reducing the running speed just before their shooting increased their shooting accuracy and that greater running speed between phases resulted in increased delay in shooting, thereby identifying some factors that can improve performance [5]. In another study, Ball et al. [6] maintained that shortening time in the shooting phase is one of the most essential determinants in improving overall laser-run performance, while stressing the necessity of training to adopt the appropriate shooting posture in the shortest time possible. However, Ball et al [6]. also noted that competitors currently rely on shooting time and accuracy, as there is still a significant lack of data with regards to the specific determinants of laser-run performance. Further systematic and scientific evidence is now required to confirm these findings.

The introduction of sports science has become a prerequisite for excellent athletic performance. In particular, advances in instrumentation for measuring and evaluating performance in sports has made it possible to develop more efficient measures for better training, thus resulting in the improvement of athletic performance [7]. Of these instruments, the respiratory gas analyzer ( $\mathrm{K} 5$, Cosmed, Italy) has made it possible to analyze changes in the physiological metabolism of athletes while they perform an outdoor sport event. Previously, respiratory gas analysis was pos- sible only for indoor. Furthermore, the application of global positioning satellites (GPS) has allowed precise evaluation of each athlete in terms of movement and performance in real time, thus improve his/her athletic performance. Dadswell et al. [3] highlighted the necessity of analyzing the determinants of athletic performance in laser-run as it is a combination of two phases that influence the final results of the entire game. Kim et al. [8], after realizing the importance of sports science, contrived to compare the performances of athletes with different levels of experience with a GPS system. However, it is thought that the location of the test described by Kim et al [8]. should have been reconsidered since the outdoor temperature ranged between -7.3 and $2^{\circ} \mathrm{C}$ and was very different from that of the actual athletic event. Consequently, the test setting was not appropriate enough to measure an athlete's metabolism or to allow athletes to perform to their full potential. Furthermore, it would be possible to evaluate the determinants that influence performance in a combined athletic event more scientifically if an athlete's metabolic changes, such as oxygen intake, respiratory quotients $(R Q)$, and respiratory frequency (RF), were assessed during the event by means of a respiratory gas analyzer, in addition to the GPS system. Generally, $\mathrm{VO}_{2} \max$ is employed as an index with which an athlete's endurance exercise capacity can be evaluated [9]. The characteristic nature of laser-run, in which an athlete should run $800 \mathrm{~m}, 4$ times, makes the assessment of $\mathrm{VO}_{2} \mathrm{max}$ through the Graded Exercise Test (G.X.T.) an important index to investigate the differences of aerobic exercise capacities between elite athletes and non-elite athletes. Such differences can be evaluated directly and the determinants of athletic performance can be identified by analyzing interrelationships between the factors involved.

This study aimed to compare groups of elite and non-elite athletes of modern pentathlon and analyze the differences between these two groups by investigating factors in performance records, such as overall laser-run event performance, running time, shooting time, and delay per shooting attempt, and by evaluating metabolic phases, such as oxygen intake, respiratory quotients, respiratory frequencies, and heart rates by employing a GPS system and an outdoor respiratory gas analyzer. These factors may provide fundamental data that could be used to develop a more efficient training strategy for this modified event.

\section{MATERIALS AND METHODS}

\section{Subjects}

The subjects were 14 modern pentathlon athletes (male) from K Uni- 
versity in South korea who had been finalists in domestic and/or international events. These athletes were divided into elite athletes $(n=7)$ and non-elite athletes $(\mathrm{n}=7)$ based on their laser-run record. All subjects were attending the same university and therefore received almost the same training. They also had similar daily routines, including sleeping and resting times, and followed a similar diet. Prior to the study, the objective and protocols were clearly explained to the prospective subjects, and only those subjects who voluntarily participated were included. All subjects provided informed written consent. The study was approved by the research ethics board of K University (20170810-010) and was performed in accordance with the Declaration of Helsinki. The characteristics of the subjects are presented in Table 1.

\section{Circumstances}

This experiment was performed on a 400-m outdoor track at K University and the performance of each athlete from the two groups was checked alternately on the same day. The day of the experiment was chosen in consideration of the fact that outdoor measurement could lead to the inclusion of some errors. The experiment was performed in appropriate circumstances for the performance of laser-run where the temperature range was $6-17^{\circ} \mathrm{C}$, wind speed was $1-2 \mathrm{~m}$, and humidity was $50 \%$. The participating subjects were requested to warm up sufficiently before the experiment.

\section{Procedures}

All subjects were made to wear sub-miniature wireless metabolism analyzers (K5, COSMED, Italy) with GPS and outdoor respiratory gas

Table 1. Characteristics of the subjects

\begin{tabular}{lrcc}
\hline & Elite $(\mathrm{n}=7)$ & Non-elite $(\mathrm{n}=7)$ & \multicolumn{1}{c}{$t$} \\
\hline Age $(\mathrm{yr})$ & $19.33 \pm 0.52$ & $19.33 \pm 0.82$ & .000 \\
Height $(\mathrm{cm})$ & $177 \pm 4.56$ & $179.67 \pm 5.28$ & -.936 \\
Weight $(\mathrm{kg})$ & $69.5 \pm 3.39$ & $72 \pm 6.03$ & -.885 \\
BMI $\left(\mathrm{kg} / \mathrm{m}^{2}\right)$ & $22.18 \pm 0.59$ & $22.27 \pm 1.1$ & -.163 \\
\hline
\end{tabular}

Values are expressed as mean \pm standard deviation.

$\mathrm{BMI}$, body mass index. analyzing functions. Each subject was allowed some time to become accustomed to wearing these analyzers prior to experimentation. The instrument used is the smallest of all market-available wireless respiratory gas analyzers and measures $16 \mathrm{~cm} \times 10 \mathrm{~cm} \times 4 \mathrm{~cm}$. Measurements were taken once, in accordance with the official rules of laser-run in the modern pentathlon; specific procedures are presented in Fig. 1. All elements were monitored in every phase and the data collected throughout the measurement were analyzed with dedicated software (Omnia, COSMED, Italy) in real time.

\section{1) Shooting phase}

The experiment provided each subject 4 shooting attempts, as required in the official laser-run event, and the subjects used the same laser-shooting system. In each shooting phase, the subjects were required to succeed in hitting 5 targets within 50 seconds before proceeding to the next phase. If they did not succeed in hitting the 5 targets within 50 seconds, the subjects proceeded to the next phase with no penalty. During the shooting phase, we calculated certain parameters: overall and average shooting accuracy over the shooting phase, shooting time, and delay per shooting attempt. In order to calculate shooting accuracy, the numbers of green signals (signifying successful shots) and red signals (signifying unsuccessful shots) were counted and recorded manually. Data relating to shooting time and delay per shooting attempt were collected in real time through the manual of expert of shooting evaluation.

\section{2) Running phase}

All participants performed while wearing the sub-miniature wireless metabolism analyzers with GPS function. The GPS feature of the subminiature wireless metabolism analyzer made it possible for us to assess the time elapsed for the entire circuit, as well as various other factors, such as elapsed running time in each section and running velocity. The respiratory gas analyzer functions also made it possible for us to assess oxygen intake, respiratory quotients, respiratory frequencies, and heart rates, thereby revealing changes in the metabolism of each athlete. Information from all factors was collected every second with the Omnia sys-

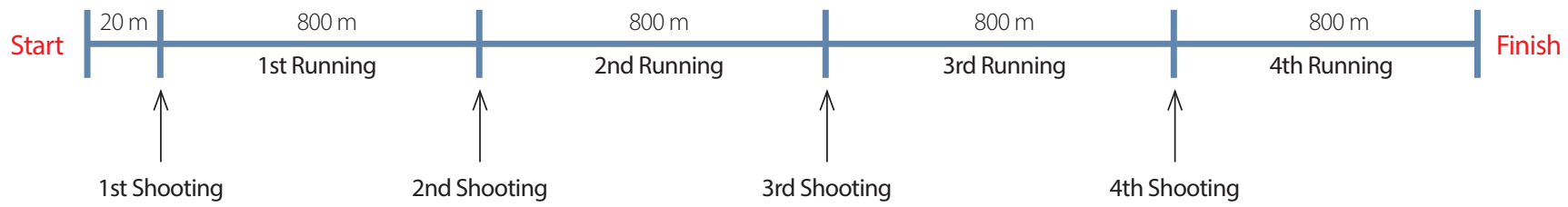

Fig. 1. Experimental design. Shooting 5 targets in a maximum of 50 seconds. 
tem (COSMED, Italy) and irregularities caused by abnormal responses were disregarded.

\section{3) Grade Exercise Test}

A treadmill (T170, COSMED, Italy), which provides almost the same running effect as laser-run, was used to assess $\mathrm{VO}_{2} \max$ and the Bruce protocol because of its wide applicability on healthy subjects to the subjects who were physically unfit $[10,11]$. The Bruce protocol is a graded exercise protocol in which an athlete is required to start at an initial velocity of $1.7 \mathrm{mph}$ on a slope with $10 \%$ elevation and the velocity is increased by $0.9 \mathrm{mph}$, and the elevation of the slope is increased by $2 \% \mathrm{ev}$ ery three minutes. Metabolic changes during the graded exercise test were assessed with the enclosed automatic respiratory gas analyzer, which evaluated the respiratory gas exhausted during the exercise. The indoor temperature range in the test room was $22-25^{\circ} \mathrm{C}$ and the subjects were prohibited from performing athletic activity or drinking alcohol for 48 hours before the test.

\section{Data processing}

All data were expressed as means \pm standard deviations using SPSS version 18.0. The independent $t$-test was conducted to further delineate between the two populations and Pearson's correlation analysis was used to determine relationships between the data. Statistical significance was set at $\alpha=.05$.

\section{RESULTS}

\section{Elapsed time for overall performance}

Elapsed time for the overall laser-run event showed that the performance in laser-run was significantly shorter in the elite group $(707.1 \pm 14.3$ second $)$ than in the non-elite group (747.18 \pm 30.45 second; $105 \%$; $p<.05$; Fig. 2).

\section{Comparison of shooting performance}

The mean delay per shooting attempt and shooting accuracy are presented in Table 2. No significant difference was observed for delay per shooting attempt between the elite group and the non-elite group $(p>.05)$; however, the non-elite group $(94.4 \pm 8.6 \%)$ showed significantly better performance over the elite group $(73.1 \pm 17.2 \%, 77 \%)$ in shooting accuracy during the first shooting phase $(p<.05)$. Total shooting time, mean shooting time, and shooting time for each phase are also presented in Table 2. No significant difference was observed for elapsed shooting time or related factors between the two groups $(p>.05)$.
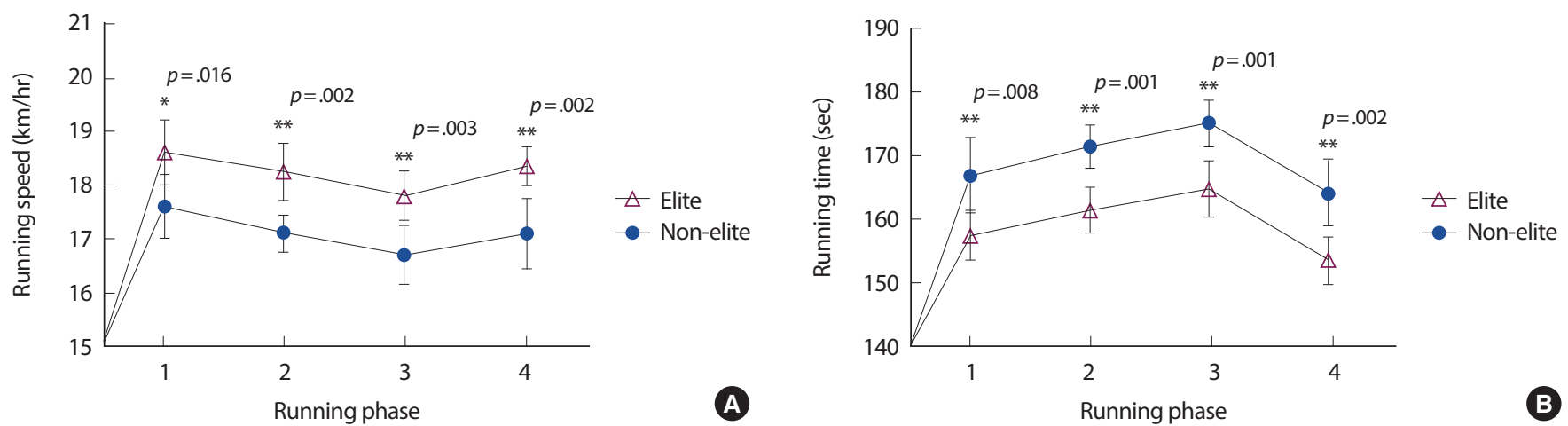

Fig. 2. Value of running (A) speed and (B) time per running phase. ${ }^{*} p<.05$ significantly different from the elite group; ${ }^{* *} p<.01$ significantly different from the elite group.

Table 2. Mean delay per shooting attempt, accuracy and time

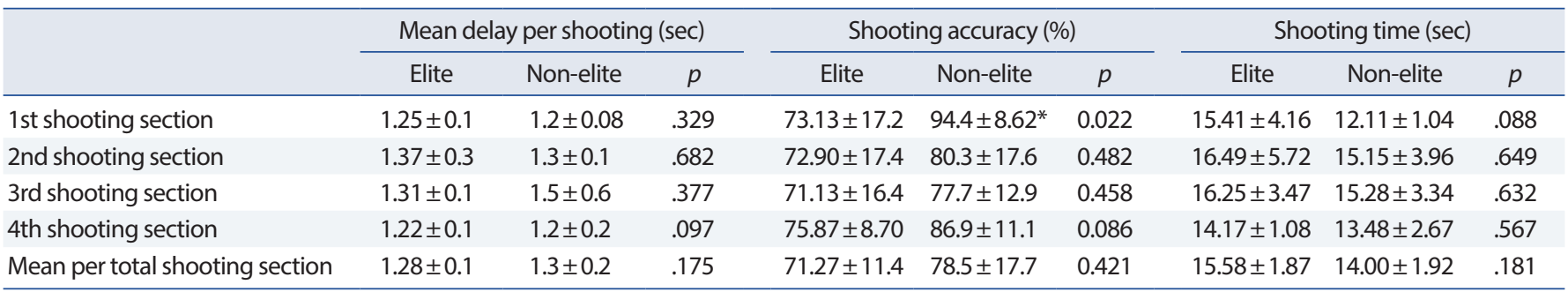

${ }^{*} p<.05$ significantly different from Elite group. 


\section{Running performance}

\section{1) Running speed}

The elite group $(18.23 \pm 0.39 \mathrm{~km} / \mathrm{h})$ showed significant excellence over the non-elite group $(17.2 \pm 0.41 \mathrm{~km} / \mathrm{h})$ in terms of running speed in each phase over the entire section $(p<.01)$. Both groups showed the greatest running speeds during the first running phase, but showed consistently reducing speed until the third phase. However, during the fourth phase, running speed recovered, reaching almost the same speed as shown in the first phase (Fig. 2A).

\section{2) Running time}

The elite group (637.28 \pm 12.79 second $)$ showed a significantly shorter running time than the non-elite group $(677.6 \pm 16.26$ second, 106\%) over the entire running section $(p<.01)$. Both groups showed an increasingly longer running time over the first three running phases, but in the fourth running phase, they shortened their running time almost to the level seen during the first running phase (Fig. 2B).

\section{3) Respiratory gas analysis results for each phase}

Respiratory gas analysis allowed us to compare metabolic changes in each group over the running phases by assessing oxygen intake RQ, RF
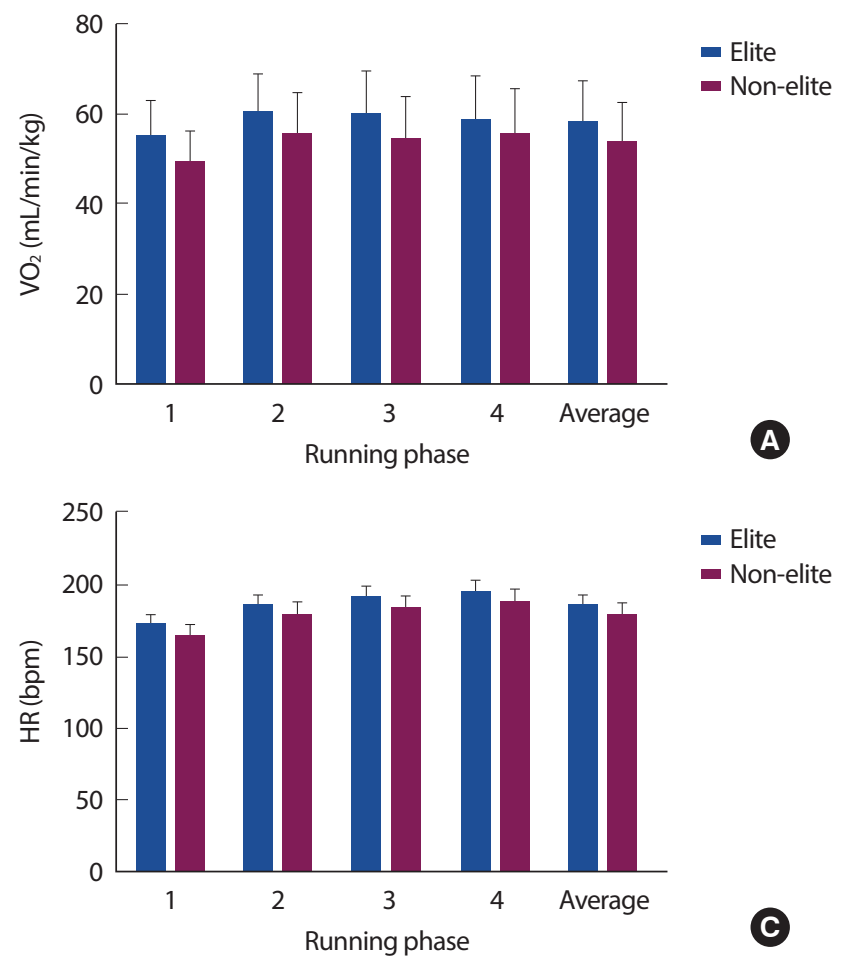

and heart rates (HR). There were no significant differences between the elite group and the non-elite group in terms of oxygen intake (Elite: 58.72 $\pm 8.88 \mathrm{~mL} / \mathrm{min} / \mathrm{kg}$, Non-elite: $53.9 \pm 8.86 \mathrm{~mL} / \mathrm{min} / \mathrm{kg}, p=.37$ ), RQ (Elite: $1.0 \pm 0.2$, Non-elite: $1.0 \pm 0.1, p=.843$ ), or HR (Elite: 186.6 \pm 7.1 , Non-elite $179.3 \pm 8.3, p=.132$ ) over the entire running phases (Fig. 3A-C). However, the elite group showed a significantly lower mean respiratory frequency $(53.93 \pm 3.83)$ than the non-elite group $(62 \pm 4.03 ; 116 \%$; $p<.05$; Fig. 3D).

\section{4) Grade exercise test}

There were no significant differences between the elite group and the non-elite group in terms of $\mathrm{VO}_{2} \max$ (Elite: $72.05 \pm 6.58 \mathrm{~mL} / \mathrm{min} / \mathrm{kg}$ Non-elite: $64.7 \pm 9.67 \mathrm{~mL} / \mathrm{min} / \mathrm{kg}, p=.06$ ), HR (Elite: $190.6 \pm 7.8$, Nonelite: $182.7 \pm 11.39, p=.191$ ) and Rf (Elite: $51.46 \pm 5.81$, Non-elite: $53.2 \pm$ $2.08, p=.509)$ follow the bruce protocol (Fig. 4A-C).

\section{Analysis of the correlations between overall performances and various factors}

To reveal the correlations between laser-run performance and associated factors, we performed Pearson's correlation analysis. Significant negative correlation was observed between overall laser-run event performance and the $\mathrm{VO}_{2} \mathrm{max}$, as evaluated using the Graded Exercise Test $(r=-.621$;


Fig. 3. Value of $(A) \mathrm{VO}_{2}$, (B) $\mathrm{RQ},(\mathrm{C}) \mathrm{HR}$, (D) Rf per running phase. $\mathrm{RQ}$, Respiratory quotient; $\mathrm{HR}$, Heart rate; Rf, Respiratory frequency. ${ }^{*} p<.05$ significantly different from the elite group. 
$p<.05$; Fig. 5A). Even though there was no significant correlation between the elapsed time over the overall laser-run event and shooting accuracy during the shooting phase $(r=-.394, p>.05)$, there was significant positive correlation in delay per shooting attempt $(r=.627 ; p<.05$; Fig. 5B,
C). Because there was a meaningful correlation between total shooting time $(r=.581, p=.047)$, total running time $(r=.899, p<.001)$, and total running speed $(r=-.908, p<.001)$ and the total elapsed time over the overall laser-run event performance, reducing the elapsed time of shooting
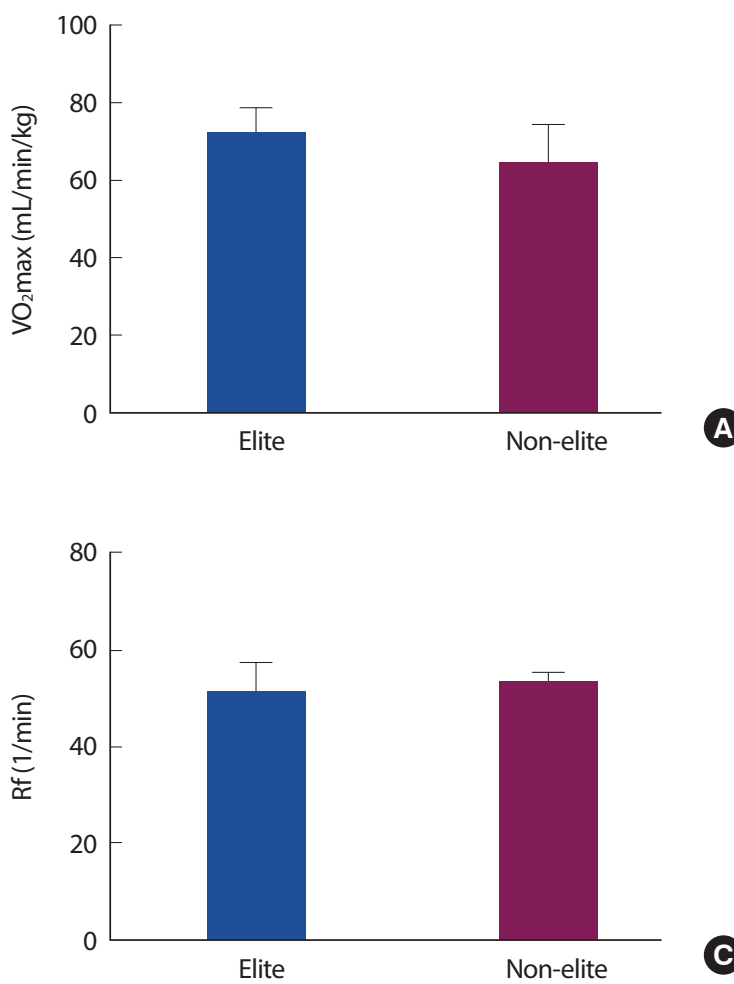

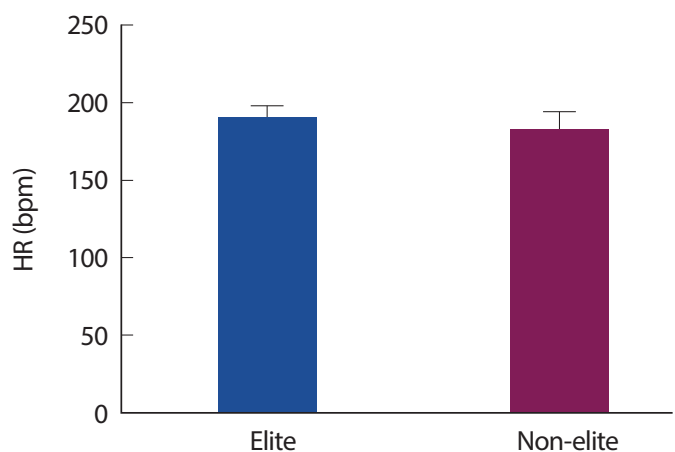

B

Fig. 4. Value of (A) $\mathrm{VO}_{2} \max$, (B) Rf, (C) HR for grade exercise test. HR, Heart rate; $\mathrm{Rf}$, Respiratory frequency.
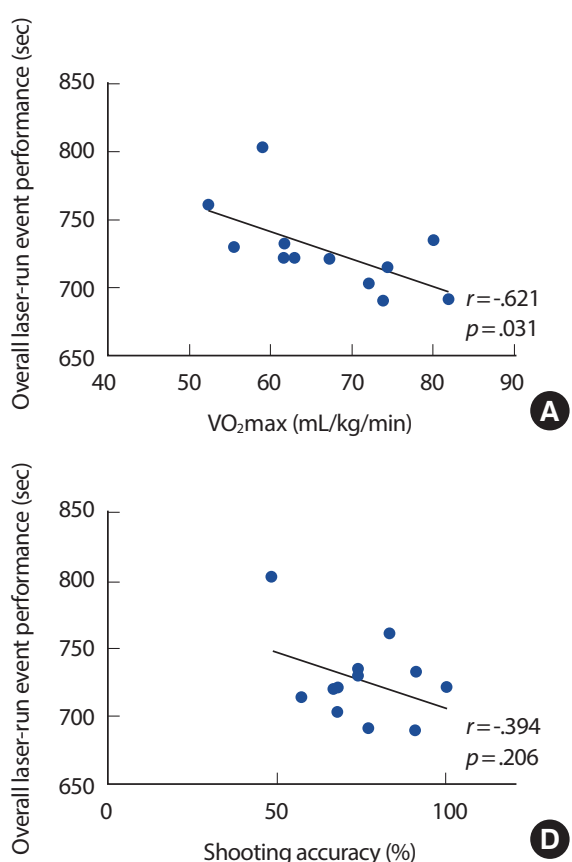

C
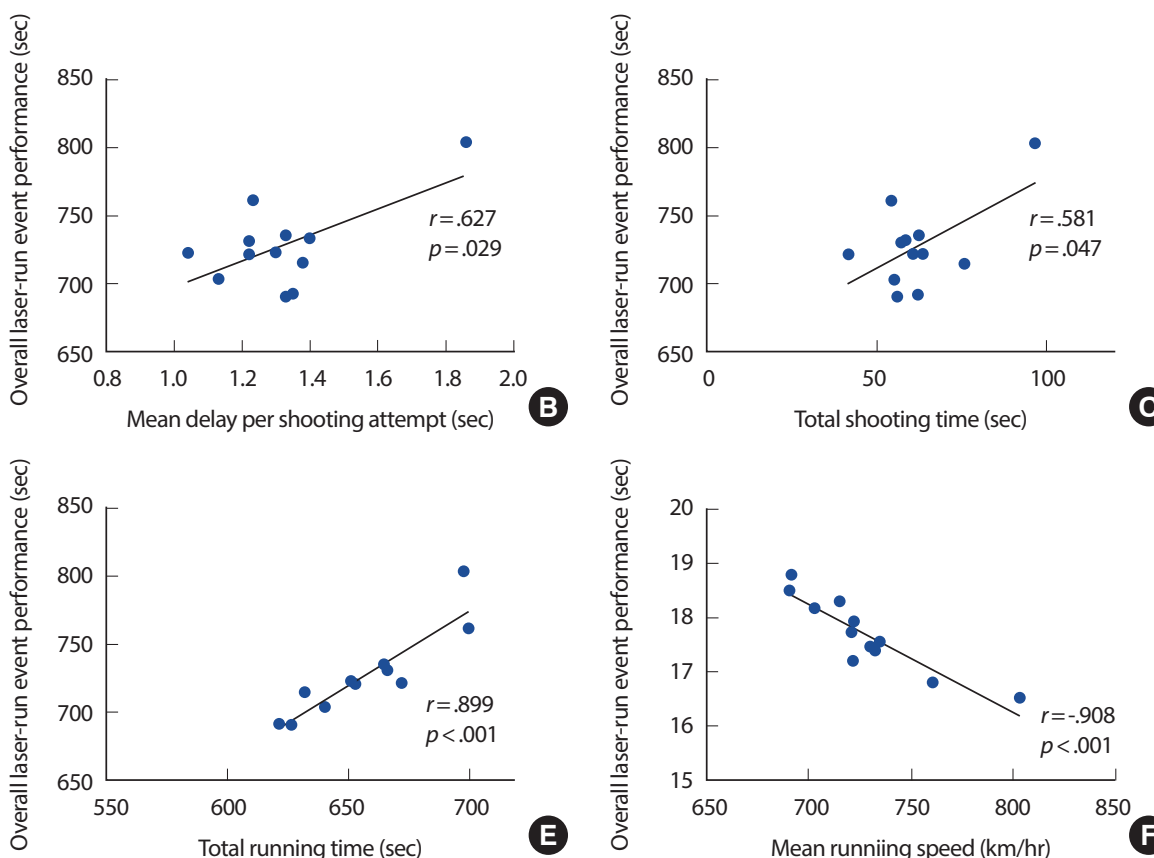

C

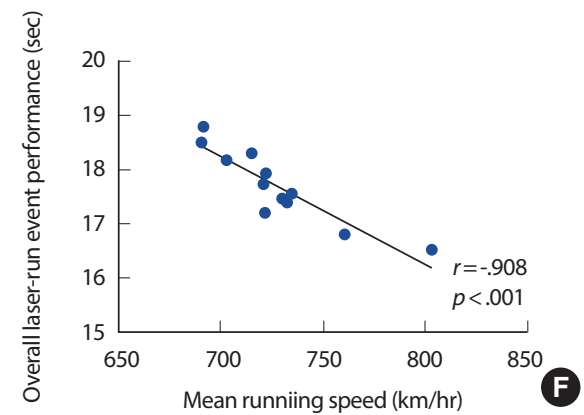

Fig. 5. Correlation between overall laser-run event performance and $(A) \mathrm{VO}_{2} \mathrm{max}$, (B) shooting accuracy, (C) mean delay per shooting attempt, (D) total shooting time, $(E)$ total running time and $(F)$ mean running speed, respectively. 
and running had a close relation to overall performance. However, we found that the elapsed time for running, and running speed, exhibited a closer relationship than shooting (Fig. 5D-F).

\section{DISCUSSION}

The aims of this study were to assess the determinants of overall laserrun event performance by dividing a cohort of modern pentathletes into groups of elite athletes and non-elite athletes and analyzing their performance in an actual laser-run event. Although the two groups did not show a significant difference in their shooting performance, the elite athletes gave a significantly better performance than the non-elite athletes in terms of running speed and running time. An analysis between elapsed time for the performance of laser-run, which is representative of an athlete's overall laser-run performance, and various factors revealed that there was a close correlation between $\mathrm{VO}_{2} \mathrm{max}$, total shooting time, shooting accuracy, delay per shooting attempt, running time, and running speed, although there were some differences in the degrees to which various factors were correlated.

In the case of laser-run in the modern pentathlon, whose rules were modified in 2017 and now require athletes to perform both shooting and running, it is essential for an athlete to identify important factors to ensure a more directed training and to garner better performance results.

The total elapsed time of performance for the elite athletes was significantly shorter than the non-elite athletes. This result showed that the rules of laser-run, which require an athlete to proceed through 4 shooting and running phases as quickly as possible, enabled the elite athletes to out-perform the non-elite athletes. Comparison between these two groups could be the basis on which we could identify determinants affecting overall performance. Le Meur et al. [5] evaluated athletes participating in the 2009 UIPM World Cup, hosted in Hungary, and reported that successful performance in laser-run depended on shooting accuracy. This precedent study, involving the analysis of upper, middle, and lower level athletes, found that shooting accuracy, rather than running time or running speed, was a meaningful determinant of successful performance. Furthermore, Dadswell et al. [3], and Goonetilleke et al. [12], suggested that for more successful laser-run event performance, an athlete should improve shooting accuracy by slowing running speed just before starting the shooting phase or by developing methods to reduce shaking, because laser-run performance is most closely related with the elapsed time during the shooting phase. However, the results of this study, unlike previous studies, did not reveal any meaningful difference between the two groups in terms of average shooting accuracy, in the total elapsed time during the shooting phase, or in the elapsed shooting time during each shooting phase (Table 2). Furthermore, in terms of shooting accuracy during each shooting phase, non-elite athletes significantly excelled elite athletes (Table 2). These results meant that negative factors such as shooting accuracy and time lapse could be offset by other factors during running or other phases.

Recently, many elite pentathletes have been concentrating on training themselves to reduce delay per shooting attempt during the shooting phases [5]. They assume that reducing the overall time taken for the 5 shots, by reducing each shooting time, even at the cost of accuracy, could represent a strategy for improving overall laser-run event performance. Ball et al. [4] also suggested the necessity of a special training regime for reducing the time taken for each shooting attempt in order to improve overall performance in laser-run. The present study also revealed that the average time difference in each shooting attempt between the fastest shooter and the slowest shooter was 0.91 seconds; thus, the time elapsed for each shooting attempt greatly affects the total time taken for the shooting phase. Therefore, reducing time in each shooting attempt, as well as improving shooting accuracy, could be a very effective way to improve overall performance in laser-run. The present study showed that there was a significant correlation among overall laser-run event performance, shooting accuracy, and elapsed time in shooting (Fig. 5). However, there was no significant difference in each shooting attempt when compared between the elite athletes and the non-elite athletes ( $\mathrm{Ta}-$ ble 2). Thus, as our correlation analysis shows, even though it is evident that each factor in the shooting phase influences overall performance in the combined event, it is not reasonable to suggest that shooting factors represent the dominant factors in determining overall performance in the event, because there was no significant difference in shooting factors between the two groups. Nowadays, almost modern pentathlon athletes have high capacity of shooting performance and max time of shooting phase is only 50 seconds. So, it is difficult for athlete to have a significantly difference of shooting benefits.

The average elapsed time for overall laser-run event performance for the elite athletes was $707.13 \pm 14.31$ seconds. The elapsed time for running (637.28 \pm 23.79 second) accounted for a time period that was 10 times as long as the elapsed time for shooting $(62.32 \pm 7.5$ second). This shows the possibility that the determinants of the running phase, rather than the time delay in the shooting phase, may have a greater influence 
upon the total elapsed time of performance in laser-run. Even though GPS assessments, using negative-split methods, showed that the two groups had the same tendency of increasing running speed in the final fourth running phase [13], the groups showed a meaningful difference in terms of running speed and elapsed time during all phases (Fig. 2). Furthermore, $\mathrm{VO}_{2} \max$ [14], the representative element of physical strength in aerobic exercise capacity, showed a significant negative correlation with overall performance in laser-run, and thus represented a potential parameter related to physical strength that could be used to anticipate overall laser-run event performance in the elite athletes (Fig. 5). Both Disch et al. [15], and Ribisl et al. [16], reported that in the running event, which covers more than $545 \mathrm{~m}$, the $\mathrm{VO}_{2}$ max had a close relationship with overall event performance. Daniel et al. also insisted that $\mathrm{VO}_{2} \mathrm{max}$ could be a reliable criterion by which overall performance in the events that require endurance could be anticipated [17]. In the present study, there were no significant differences between the elite and non-elite athletes in terms of oxygen intake, RQ, or HR in any of the running phases (Fig. 3A-C). However, the elite athletes showed a lower Rf than the nonelite athletes (Fig. 3D). The capability of having a lower Rf in the same exercise intensity implies excellence in terms of oxygen utilization rate in the muscle fibers during endurance exercise and thus promises a positive influence upon oxygen intake and ventilation volume [18]. Consequently, the findings of this study suggested that unlike factors in the shooting phase, in which there was no meaningful difference between the elite and non-elite athletes, running speed, running time, and mean respiratory frequencies during the running phase showed a significant difference between the two groups and that the $\mathrm{VO}_{2}$ max had a close correlation with, and could therefore be an important determinant of, overall laser-run performance.

Overall performance in laser-run showed a positive correlation with shooting and elapsed time during the running phase (Fig. 5). Therefore, it is important to reduce the elapsed time during shooting and running in order to improve overall performance in laser-run. However, our analysis of overall laser-run event performance, and factors related to both the running and shooting phases revealed that overall laser-run event performance had a closer correlation with elapsed time in running $(r=.899$, $p<.001)$ and running speed $(r=.908, p<.001)$ rather than with elapsed time during shooting $(r=.581, p=.047)$. This suggested that elapsed time during running could be a more important determinant for overall higher performance ability in laser-run.

\section{CONCLUSION}

In conclusion, the rules of laser-run in modern pentathlon were modified in 2009, thus necessitating an athlete to perform both shooting with a laser pistol and running in a combined manner. Therefore, there is an urgent need to identify the factors that could determine overall performance in laser-run. In this event, it is essential for athletes to train themselves in all related elements of physical strength, since overall event performance is closely related with a variety of factors associated with shooting and running. However, one of the most important factors in determining the result of laser-run are those elements of physical strength that could reduce elapsed running time. Thus, it is important that modern pentathletes concentrate on training programs that aim to improve cardiovascular endurance. This could reduce the elapsed time in the running phase and represent the most effective way to improve their overall event performance.

One question that remains is whether the results of this research can be applied to the entire population of competitors, from top international modern pentathletes to amateurs. However, the top internationals involved with the study of Le Meur et al. [5] showed a similar distribution in records. Furthermore, the overall performance of domestic modern pentathletes has already reached world-class standard. This shows clearly that improving cardiovascular endurance and muscular endurance to reduce elapsed time during the running phase is a powerful determinant for a superlative performance. Furthermore, it is highly desirable for future research to analyze overall laser-run event performance by taking advantage of advancements in high-technology sports science. We hope that the data from this present study will be helpful in developing training programs to improve overall laser-run event performance.

\section{REFERENCES}

1. Jeong CS. Characteristics of performance-related physical fitness and physique in elite modern pentathlon players [thesis]. Seoul: Korea National University 2006.

2. Kelm J, Modern Pentathlon, in Epidemiology of Injury in Olympic Sports, Wiley-Blackwell 2009. p. 176-180.

3. Dadswell C, Payton C, Holmes P, Burden A. The effect of time constraints and running phases on combined event pistol shooting performance. J Sports Sci. 2016;34(11):1044-50.

4. Le Meur Y, Dorel S, Baup Y, Guyomarch JP, Roudaut C, et al. Physio- 
logical demand and pacing strategy during the new combined event in elite pentathletes. Eur J Appl Physiol. 2012;112(7):2583-93.

5. Le Meur Y, Hausswirth C, Abbiss C, Baup Y, Dorel S. Performance factors in the new combined event of modern pentathlon. J Sports Sci. 2010;28(10):1111-6.

6. Ball KA, Best RJ, Wrigley TV. Body sway, aim point fluctuation and performance in rifle shooters: inter- and intra-individual analysis. J Sports Sci. 2003;21(7):559-66.

7. Jennings D, Cormack SJ, Coutts AJ, Aughey RJ. GPS analysis of an international field hockey tournament. Int J Sports Physiol Perform. 2012;7(3):224-31.

8. Kim YS, Yoon JR, Hur S. Comparison of new combined event performance by performance level in modern pentathlon athletes. Exerc Sci. 2016;25(4):282-7.

9. Tjonna AE, Leinan IM, Bartnes AT, Jenssen BM, Gibala MJ, et al. Lowand high-volume of intensive endurance training significantly improves maximal oxygen uptake after 10-weeks of training in healthy men. PLoS One. 2013;8(5):e65382.

10. Bruce RA, Kusumi F, Hosmer D. Maximal oxygen intake and nomographic assessment of functional aerobic impairment in cardiovascular disease. Am Heart J. 1973;85(4):546-62.

11. Thompson PD, Arena R, Riebe D, Pescatello LS. ACSM's new prepar- ticipation health screening recommendations from ACSM's guidelines for exercise testing and prescription, ninth edition. Curr Sports Med Rep. 2013;12(4):215-7.

12. Goonetilleke RS, Hoffmann ER, Lau WC. Pistol shooting accuracy as dependent on experience, eyes being opened and available viewing time. Appl Ergon. 2009;40(3):500-8.

13. Abbiss CR, Laursen PB. Describing and understanding pacing strategies during athletic competition. Sports Med. 2008;38(3):239-52.

14. Astrand PO, Saltin B. Maximal oxygen uptake and heart rate in various types of muscular activity. J Appl Physiol. 1961;16:977-81.

15. Disch J, Frankiewicz R, Jackson A. Construct validation of distance run tests. Res Q Exerc Sport. 1975;46(2):169-76.

16. Ribisl PM, Kachadorian WA. Maximal oxygen intake prediction in young and middle-aged males. J Sports Med Phys Fitness. 1969;9(1): $17-22$.

17. Daniels J, Daniels N. Running economy of elite male and elite female runners. Medicin \& Science in Sports \& Exercise. 1992;24(4):483-9.

18. Miki K, Maekura R, Kitada S, Miki M, Yoshimura K, et al. Pulmonary rehabilitation for COPD improves exercise time rather than exercise tolerance: effects and mechanisms. Int J Chron Obstruct Pulmon Dis. 2017;12:1061-70. 